\title{
Evolution and Functional Insights of Different Ancestral Orthologous Clades of Chitin Synthase Genes in the Fungal Tree of Life
}

\section{OPEN ACCESS}

Edited by:

Thiago Motta Venancio, Universidade Estadual do Norte

Fluminense, Brazil

Reviewed by:

Lakshminarayan M. lyer,

National Center for Biotechnology

Information, National Library of Medicine, National Institutes of Health

USA

Robson Francisco De Souza, Universidade de São Paulo, Brazil A. Maxwell Burroughs,

National Center for Biotechnology

Information, National Library of Medicine, National Institutes of Health,

USA

${ }^{*}$ Correspondence:

Huiquan Liu

liuhuiquan@nwsuaf.edu.cn

${ }^{\dagger}$ These authors have contributed equally to this work.

Specialty section

This article was submitted to Bioinformatics and Computational

Biology,

a section of the journal

Frontiers in Plant Science

Received: 11 September 2015

Accepted: 11 January 2016

Published: 01 February 2016

Citation:

Li M, Jiang C, Wang Q, Zhao Z, Jin Q, $X u$ J-R and Liu H (2016) Evolution and Functional Insights of Different

Ancestral Orthologous Clades of

Chitin Synthase Genes in the Fungal

Tree of Life. Front. Plant Sci. 7:37.

doi: 10.3389/fpls.2016.00037

\author{
Mu $\mathrm{Li}^{1 \dagger}$, Cong Jiang ${ }^{1 \dagger}$, Qinhu Wang ${ }^{1}$, Zhongtao Zhao ${ }^{2}$, Qiaojun $\mathrm{Jin}^{1}$, Jin-Rong $\mathrm{Xu}^{1,3}$ and \\ Huiquan Liu ${ }^{1 *}$
}

${ }^{1}$ State Key Laboratory of Crop Stress Biology for Arid Areas, College of Plant Protection, Northwest A\&F University, Yangling, China, ${ }^{2}$ South China Botanical Garden, Chinese Academy of Sciences, Guangzhou, China, ${ }^{3}$ Department of Botany and Plant Pathology, Purdue University, West Lafayette, IN, USA

Chitin synthases (CHSs) are key enzymes in the biosynthesis of chitin, an important structural component of fungal cell walls that can trigger innate immune responses in host plants and animals. Members of $\mathrm{CHS}$ gene family perform various functions in fungal cellular processes. Previous studies focused primarily on classifying diverse CHSs into different classes, regardless of their functional diversification, or on characterizing their functions in individual fungal species. A complete and systematic comparative analysis of $\mathrm{CHS}$ genes based on their orthologous relationships will be valuable for elucidating the evolution and functions of different CHS genes in fungi. Here, we identified and compared members of the CHS gene family across the fungal tree of life, including 18 divergent fungal lineages. Phylogenetic analysis revealed that the fungal $\mathrm{CHS}$ gene family is comprised of at least 10 ancestral orthologous clades, which have undergone multiple independent duplications and losses in different fungal lineages during evolution. Interestingly, one of these CHS clades (class III) was expanded in plant or animal pathogenic fungi belonging to different fungal lineages. Two clades (classes $\mathrm{Vlb}$ and $V I c$ ) identified for the first time in this study occurred mainly in plant pathogenic fungi from Sordariomycetes and Dothideomycetes. Moreover, members of classes III and $\mathrm{VIb}$ were specifically up-regulated during plant infection, suggesting important roles in pathogenesis. In addition, CHS-associated networks conserved among plant pathogenic fungi are involved in various biological processes, including sexual reproduction and plant infection. We also identified specificity-determining sites, many of which are located at or adjacent to important structural and functional sites that are potentially responsible for functional divergence of different CHS classes. Overall, our results provide new insights into the evolution and function of members of $\mathrm{CHS}$ gene family in the fungal kingdom. Specificity-determining sites identified here may be attractive targets for further structural and experimental studies.

Keywords: chitin synthase, fungi, evolution, functional diversification, plant infection 


\section{INTRODUCTION}

Chitin, a polymer of $N$-acetylglucosamine, is the second most abundant biomass in nature after cellulose (Merzendorfer, 2011). It is an important structural component of the cell wall in fungi, playing a crucial role in the maintenance of cell morphology (Bowman and Free, 2006). As a classic pathogen-associated molecular pattern (PAMP), fungal chitin can trigger innate immune responses in host plants and animals (Shibuya and Minami, 2001; Reese et al., 2007). A number of enzymes participate in the formation of chitin, but the chitin synthases (CHSs) are the crux of this process. CHSs are located in the plasma membrane and transfer $N$-acetylglucosamine to growing chitin chains (Merzendorfer, 2011). Owing to the lack of chitin in plants and mammals, CHSs are considered attractive targets for developing efficient antifungal agents used to control pathogenic fungi (Free, 2013).

CHS genes have been identified in various fungi. According to the phylogenetic positions and similarity in domain architecture, these genes were divided into three divisions (Roncero, 2002; Choquer et al., 2004; Mandel et al., 2006; Latgé, 2007). CHSs in division I contain type I (CS1, PF01644) and type II (CS2, PF03142) chitin synthase domains, as well as a chitin synthase N-terminal domain (CS1N, PF08407); CHSs in division II contain CS2 and other additional domains; CHSs in division III contain only the CS2 domain (Choquer et al., 2004; Mandel et al., 2006). Division I was further divided into three classes I, II, and III, and division II comprised three classes IV, V, and VII, whereas division III contained the single class VI (Mandel et al., 2006). Previous studies focused primarily on classifying diverse CHSs into different classes (Choquer et al., 2004; Mandel et al., 2006; Ruiz-Herrera and Ortiz-Castellanos, 2010). The majority of these studies employed a limited number of fungal species from a narrow range of fungal lineages (mostly later-branching fungi), which could lead to difficulty in establishing the relationships among different classes and even ambiguously classifying some CHSs into different or multiple classes. For example, class VI was clustered with division I in the study by Odenbach et al. (2009) but with division II in the study by Niño-Vega et al. (2004). In addition, some CHS genes from basidiomycetes of class II in the study of Ruiz-Herrera and Ortiz-Castellanos (2010) were classified into classes I and II in the study of Munro and Gow (2001). More recently, Pacheco-Arjona and Ramirez-Prado classified CHSs from 54 fungal genomes into the existing classification scheme (Pacheco-Arjona and RamirezPrado, 2014). However, the established classes of CHSs may contain distant ancestral paralogs with distinct functions in fungi. Because the orthologs retain the same function (Koonin, 2005), evolutionary classification of CHS genes based on the orthologous relationship is more reliable for transferring functional annotation.

Different CHSs have been proved to play various roles, suggesting that members of this family have undergone functional diversification to a certain extent. For example, the budding yeast Saccharomyces cerevisiae has three CHS genes with different functions. ScCHS1 replenishes chitin in the cell wall of daughter cells after cell division as a repair enzyme (Cabib et al., 1989). ScCHS2 participates in the processes of primary septum formation and cell division (Silverman et al., 1988). The majority of chitin is synthesized by ScCHS3, which is also responsible for forming the ring of chitin at the budding site (Shaw et al., 1991; Schmidt, 2004). Roles of CHSs are more complicated in filamentous fungi. In the plant pathogenic fungus Magnaporthe oryzae, for example, CHSs also participate in processes related to pathogenicity. The Mochs 1 deletion mutant has a defect in conidiogenesis, which limits the infection of host plants (Kong et al., 2012). MoCHS7 is responsible for appressorium penetration and invasive growth (Kong et al., 2012). Previous studies focused primarily on the functions of CHSs in individual fungal species. A complete and systematic comparative analysis of CHS gene family across the fungal kingdom will be valuable for elucidating their evolutionary and functional relationships.

In this study, we systematically identified members of the CHS gene family across 109 representative fungi from 18 major fungal lineages. The orthologous and paralogous relationships among various CHS genes were resolved. We found that one orthologous clade of CHS genes (class III) was expanded mainly in important animal or plant pathogenic fungi from different fungal lineages. We also report the identification of two novel clades of CHSs (classes VI $b$ and VIc) that are present mainly in pathogenic fungi from Sordariomycetes and Dothideomycetes. Further expression analyses showed that members of classes III and VI $b$ were specifically upregulated during plant infection, suggesting important roles in pathogenesis. Moreover, we found that CHS-associated networks are conserved in plant pathogenic fungi and involved in various biological processes, including sexual reproduction and plant infection. In addition, we determined the specificitydetermining sites that are potentially responsible for functional diversification of different CHS genes. Our results provide new insights into the evolution and function of the fungal CHS genes.

\section{RESULTS AND DISCUSSION}

\section{The Fungal CHS Gene Family is Composed of at Least 10 Ancestral Orthologous Clades}

Based on the common conserved domains of CS1 and CS2, we identified $978 \mathrm{CHS}$ genes in the predicted proteomes of 109 representative fungi from 18 fungal lineages in phyla Cryptomycota, Microsporidia, Neocallimastigomycota, Chytridiomycota, Entomophthoramycotina, Zygomycota, Glomeromycota, Ascomycota, and Basidiomycota (Figure 1; Table S1). Among these, 373 genes contained CS1N, CS1, and CS2 domains, suggesting that these genes were members of division I. Five additional genes lacked the CS2 domain and one of those also lacked the CS1N domain, which may be due to genome assembly gaps. The remaining 600 genes harbored only the CS2 domain, suggesting that these genes are members of division II or III. 


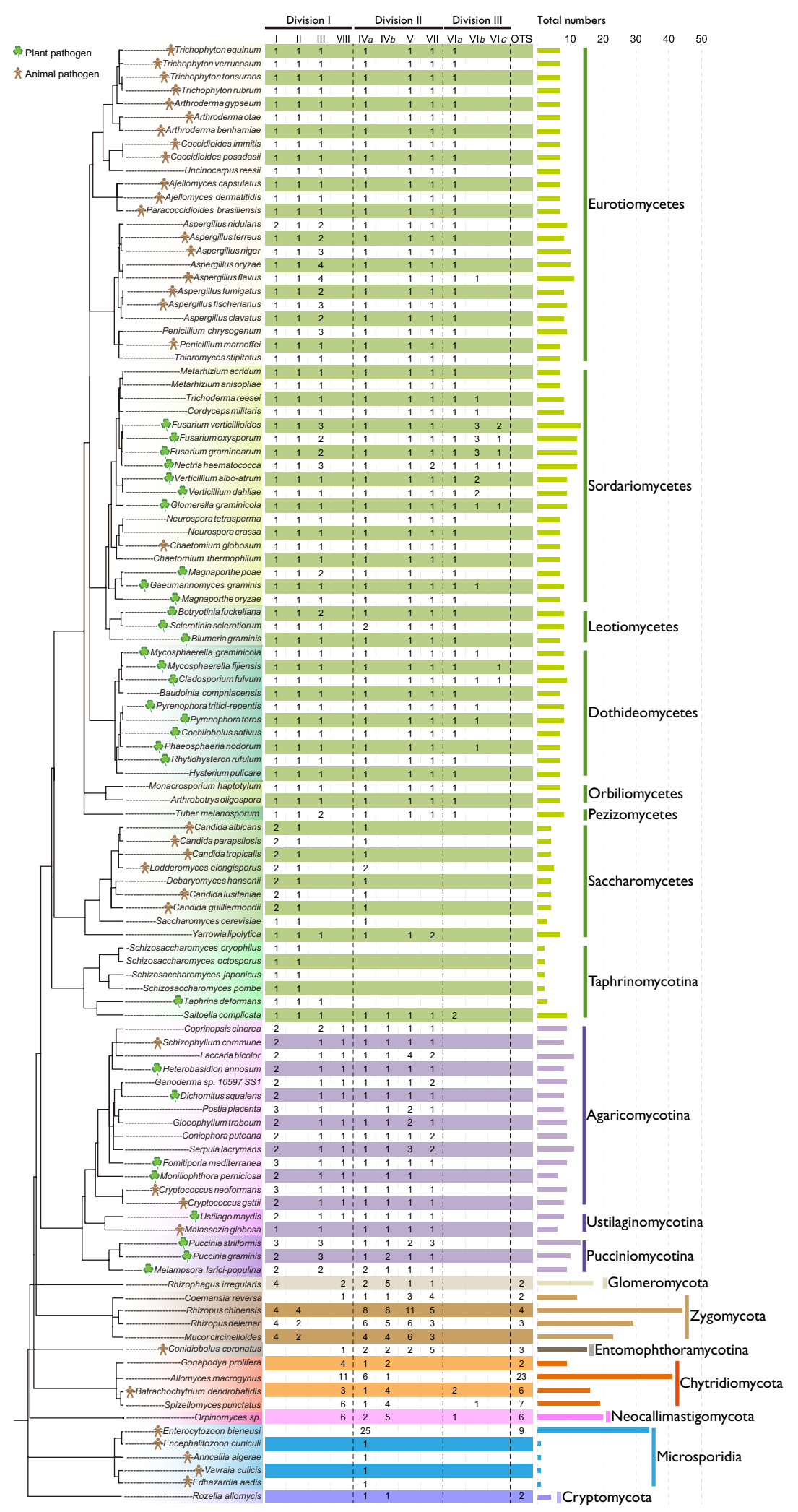

FIGURE 1 | Distribution of CHS genes across different fungal lineages. The copy number of each orthologous CHS class is indicated. The species tree was constructed based on the phylogenetic tree of $\alpha$-tubulins. The horizontal bar indicates the total number of CHSs in each fungus. For the definition of I, II, III, IVa, IVb, V, VII, Vla, VIb, VIc, VIII, and others (OTS), please see the text. 
We then performed maximum likelihood phylogenetic analyses to determine the orthologous and paralogous relationships of these identified CHS genes based on their common conserved domains. The phylogenetic tree of division I displayed four distinct ancestral orthologous clades (Figures 2,4A). Three of these orthologous clades correspond to classes I, II and III defined previously (Munro and Gow, 2001; Choquer et al., 2004). Therefore, we define them as classes I, II and III, respectively. Notably, previous studies showed ambiguous phylogenetic positions of classes I and II CHS genes from basidiomycetes (Munro and Gow, 2001; Pacheco-Arjona and Ramirez-Prado, 2014), but our study clearly suggested that these CHS genes should be within class I. A fourth orthologous clade contained CHS genes from basidiomycetes and early-branching fungal lineages, including Neocallimastigomycota, Chytridiomycota, Zygomycota, and Glomeromycota. This clade was identified for the first time in this study and defined as class VIII.

Phylogenetic analysis showed that members of divisions II and III are distantly related to each other (Figures 3,4B). Division II is comprised of four distinct orthologous clades. Two clades correspond to classes V and VII defined previously (Mandel et al., 2006) and are designated as classes V and VII in this study. Notably, class IV defined previously (Munro and Gow, 2001; Choquer et al., 2004; Pacheco-Arjona and Ramirez-Prado, 2014) was actually comprised of two orthologous clades: classes IV $a$ and IV $b$. Orthologous relationships of some CHS genes from early-branching fungi were not determined. These genes

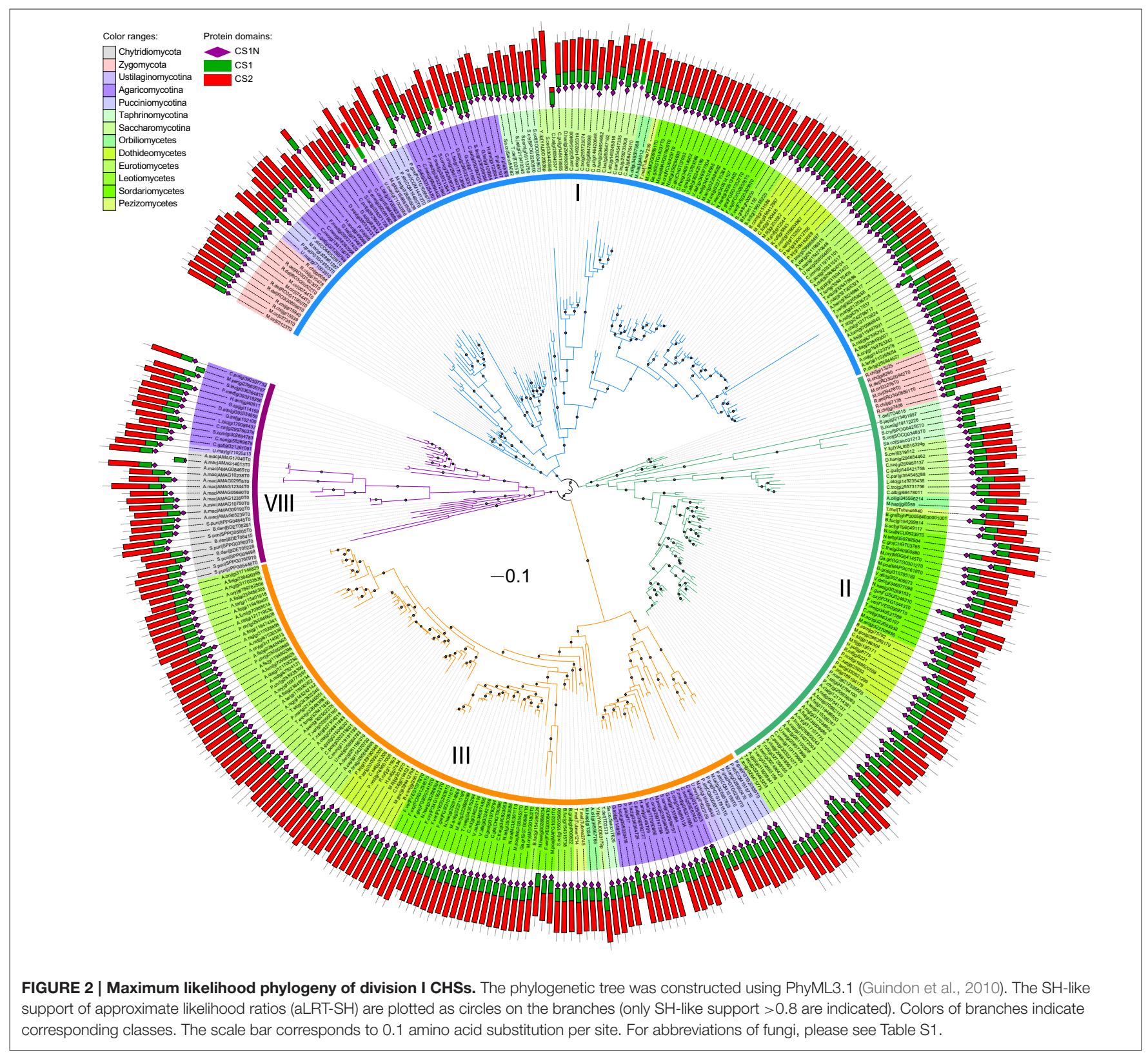




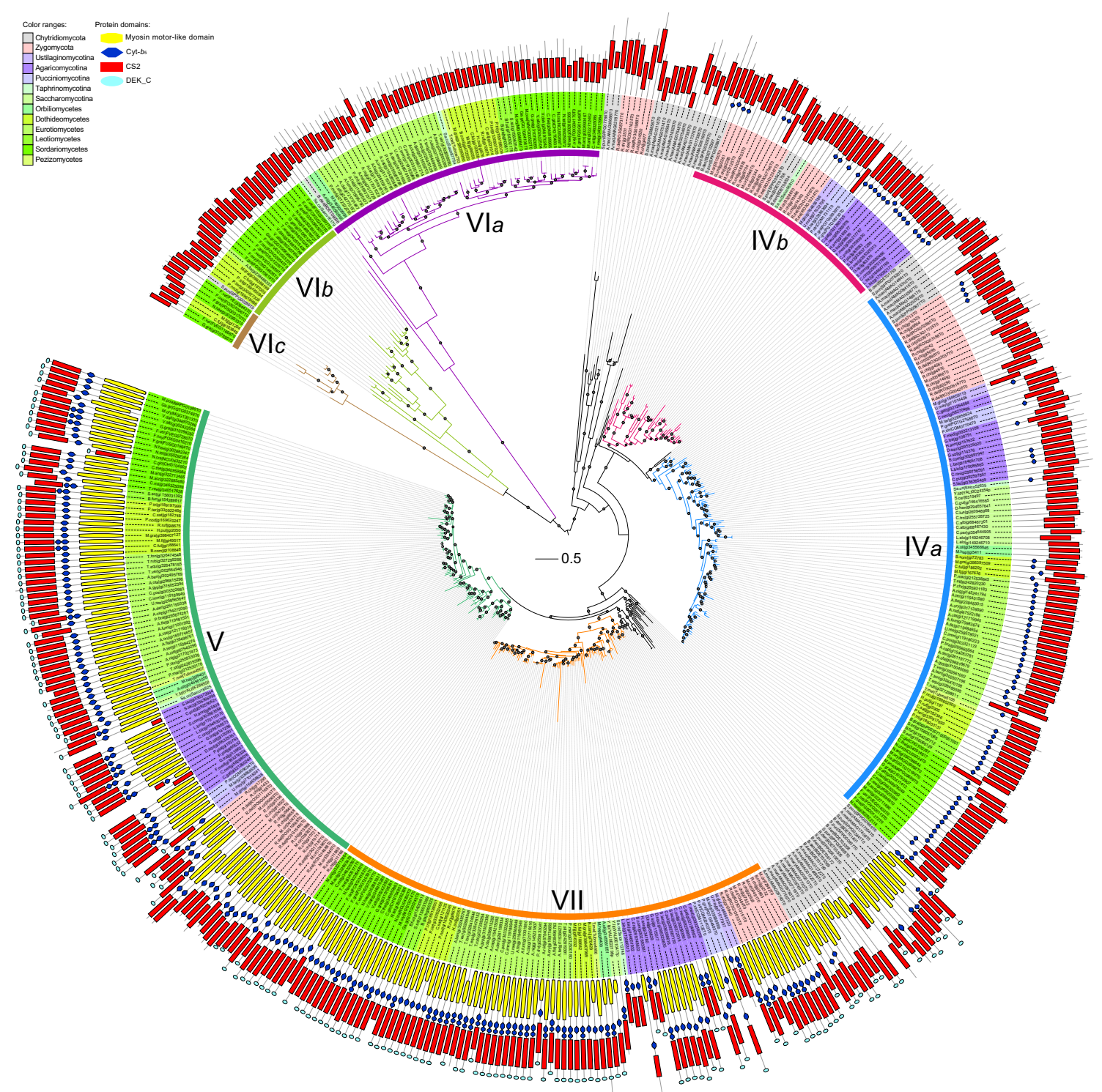

FIGURE 3 | Maximum likelihood phylogeny of divisions II and III CHSs. The phylogenetic tree was constructed using PhyML3.1. The SH-like support of approximate likelihood ratios (aLRT-SH) are plotted as circles on the branches (only SH-like support $>0.8$ are indicated). Colors of branches indicate corresponding classes. The scale bar corresponds to 0.5 amino acid substitution per site. For abbreviations of fungi, please see Table S1.

were temporarily labeled as "others" (OTS) (Figure 1). Division III is comprised of three distinct orthologous clades. One of them (class VIa) corresponds to class VI defined previously (Munro and Gow, 2001). The other two orthologous clades (classes $\mathrm{VI} b$ and $\mathrm{VI} c$ ) are reported for the first time in this study.

Comparison of sequence identity of CHSs showed that members in the same class are more closely related to each other, but distantly related to members in other classes (Figure S1). Each class formed a distinct group. These results are consistent with those of our phylogenetic analysis. CHSs of division III are more similar to those of division II than division I.

\section{Domain Architectures of CHSs are Consistent in Divisions I and III but Diverse in Division II}

Besides the three CS1N, CS1, and CS2 domains, no additional domains were identified in CHSs of division I (Figure 2). Likewise, no other domain was found in CHSs of division III besides the CS2 domain, but most CHSs of division II also contain the Cytochrome b5-like (Cyt- $b_{5}$, PF00173) domain (Figure 3). This domain is absent from some members of classes IV $a$ and IV $b$ in division II. The Cyt- $b_{5}$ domain can bind heme and steroids in electron transfer (Schenkman and Jansson, 2003), but the ligand for this domain in fungal CHSs is unknown. An 


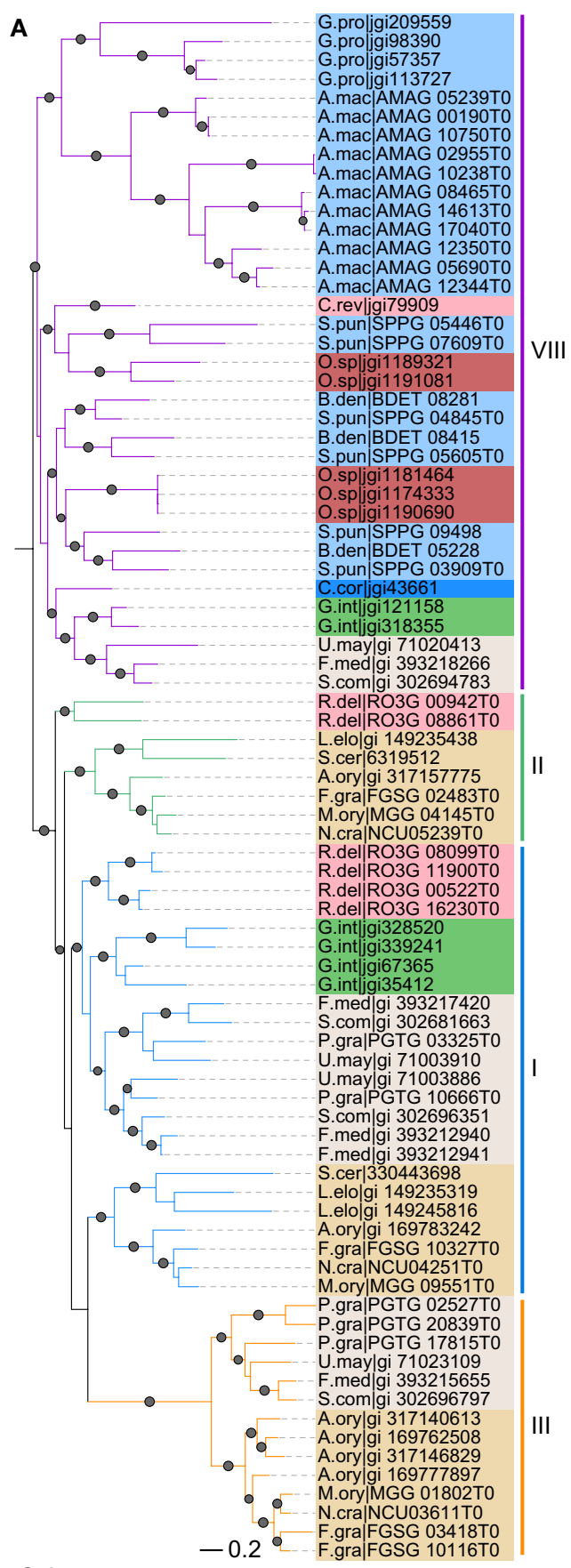

B

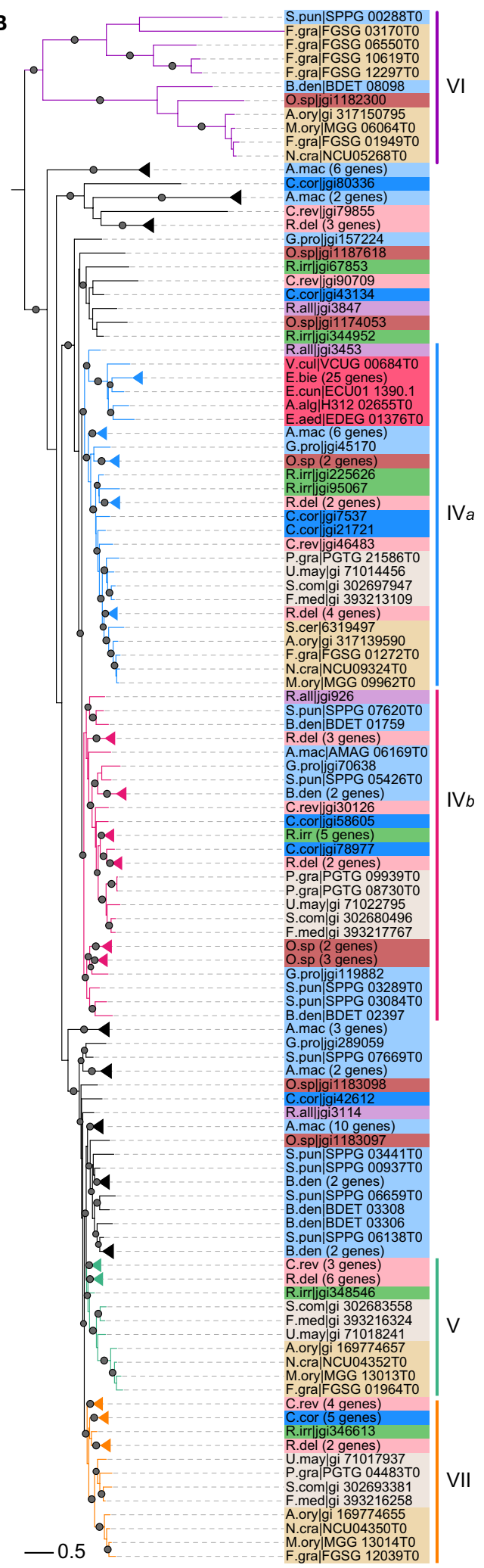

FIGURE 4 | Maximum likelihood phylogenies of CHS genes from early-branching fungi. (A) Division I. (B) Divisions II and III. Phylogenetic tree was constructed using PhyML3.1. The SH-like support of approximate likelihood ratios (aLRT-SH) are plotted as circles on the branches (only SH-like support $>0.8$ are indicated). Colors of branches indicate corresponding classes. For abbreviations of fungi, please see Table S1. 
N-terminal myosin motor-like domain (MMD, PF00063) and a DEK C-terminal (DEK_C, PF08766) domain also exist in classes $\mathrm{V}$ and VII CHSs. The MMD assists the localization of CHSs to the hyphae via interaction with the actin cytoskeleton, and facilitates polarized exocytosis (Tsuizaki et al., 2009; Schuster et al., 2012). The DEK_C domain is related to the C-terminal of animal DEK protein, which is responsible for self-multimerization and DNA binding (Kappes et al., 2004). Nevertheless, the role of the DEK_C domain in fungi remains to be determined.

\section{Variable Numbers of CHSs in Different Fungal Lineages}

Yeasts generally contain few CHSs (Figure 1). For example, the fission yeasts possess only two CHSs, the fewest copies among all fungal lineages, which may be related to the absence of chitin in their vegetative cell walls (Free, 2013). The budding yeast $S$. cerevisiae harbors three CHSs. Filamentous fungi usually contain a larger number of CHSs. For example, the cereal pathogen Fusarium verticillioides has $13 \mathrm{CHSs}$, the highest number of copies among the later-branching fungi, ascomycetes and basidiomycetes. On the other hand, the early-branching fungi, chytridiomycetes, entomophthoromycetes, zygomycetes, and glomeromycetes, generally possess more CHSs than the later-branching fungi (Figure 1), likely because the cell walls of early-branching fungi contain a higher percentage of chitin than those of later-branching fungi (Ma et al., 2009). Unexpectedly, the zygomycete Rhizopus chinensis has 44 CHS genes, the largest number of CHS genes discovered to date. Microsporidia generally contain one CHSs, but Enterocytozoon bieneusi has many more CHS genes than other species in this lineage, consistent with a previous report (Pacheco-Arjona and RamirezPrado, 2014).

\section{Frequent Duplications and Losses of CHS Genes in Different Fungal Lineages}

The classes IV $a, \mathrm{IV} b, \mathrm{VI} a, \mathrm{VI} b$, and VIII occur in both earlybranching fungi and later-branching fungi (Figure 1), suggesting that they were generated in fungal ancestors. However, during subsequent evolution, the class VIII was lost in all ascomycetes and most zygomycetes. In addition, the class III was lost in all early-branching fungal lineages, in agreement with previous reports (Ruiz-Herrera and Ortiz-Castellanos, 2010; PachecoArjona and Ramirez-Prado, 2014). The class IVb was lost in all ascomycetes but not in the archiascomycetous yeast Saitoella complicata. The class IVa was lost in individual fungal lineages, such as fission yeasts. The classes VI $a$ and VI $b$ were lost in basidiomycetes and most of early-branching fungi. Unexpectedly, in contrast to other yeasts that retained only the classes I, II, and IVa, Yarrowia lipolytica and S. complicata contained most of the orthologous classes.

In addition to gene losses, duplications of the CHS genes occurred frequently in different fungal lineages during evolution. For example, members of class I were duplicated in Basidiomycota, Zygomycota, and Candida species. CHS genes in each class were duplicated multiple times in earlybranching fungi, and those from one species generally clustered together (Figures 2-4), suggesting that they were derived from species-specific expansions. In fact, the genome of Rhizopus delemar underwent a whole-genome duplication and recent gene duplications (Ma et al., 2009).

Notably, duplications in members of class III were most common in plant or animal pathogens within Aspergillus spp., Fusarium spp., and Pucciniomycotina. Since the class III CHS has been experimentally demonstrated to play important roles during host infection in the rice blast fungus $M$. oryzae (Kong et al., 2012), the expansion of this class in pathogenic fungi may be related to infection and pathogenicity of these fungi. Furthermore, the classes VI $b$ and VIc CHSs occurred mainly in plant pathogens from Sordariomycetes and Dothideomycetes.

\section{Specificity-Determining Sites Responsible for Functional Divergence in the CHS Gene Family}

Previous studies have revealed that CHSs of several classes perform different functions in fungi (Silverman et al., 1988; Cabib et al., 1989; Shaw et al., 1991; Kong et al., 2012). It is known that functional divergence commonly results from the shifts at specificity-determining sites (Gu, 2001). Therefore, we examined CS domains among the orthologous classes for functional specificity-determining sites.

In division I, we identified 15 specificity-determining sites (Figure 5). Four of them are likely related to type I functional divergence. Residues at these sites are conserved in one orthologous clade but highly variable in the other clades, indicating that functional constraints have changed between them (Gu, 2001). The remaining 11 sites are related to type II functional divergence. Residues at these sites are conserved in different clades but their properties are dissimilar, which could lead to functional specifications within a gene family (Gu, 2001). Ten specificity-determining sites are potentially responsible for the functional divergence between class III and the other three classes. In addition, the residue at site 180 is Phe (F) in classes III and VIII, while the corresponding residue in classes I and II is Tyr (Y). Functional divergence of class II from the other three classes may be due to a shift at site 460 from Ser (S) to Ala (A). In division II, we identified 12 type II specificitydetermining sites. Residues at all of these sites in classes IV $a$ and IV $b$ are distinct from those in classes V and VII. At site 123, functional divergence of class IV a from class IV $b$ may be due to a shift from His $(\mathrm{H})$ to Phe $(\mathrm{F})$. At site 344, functional divergence of class $\mathrm{V}$ from class VII may result from a Ser (S) to His $(\mathrm{H})$ shift. In division III, we identified four type II specificity-determining sites. Residues at these sites differ among the three classes. For example, the residues at site 209 are Cys (C), Thr (T), and Ser (S) in classes VI $a, \mathrm{VI} b$, and VIc, respectively.

Most of the specificity-determining sites are adjacent to or located at the functionally or structurally important sites of CHSs identified by ConSurf (Figure 5; Figures S2-S4; Ashkenazy et al., 2010). For example, in division I, type II specificitydetermining site 80 is directly involved in ligand binding (Figure 5). This shift probably affects the chemical conformation 
of CHSs. Additionally, in division II, the structural site 346, which may have undergone type II functional divergence from division I, is adjacent to the acceptor saccharide binding sites. This shift may affect the interaction between CHSs and acceptors. Previous studies have experimentally demonstrated two functionally important motifs EDRXL and QRRRW of CHSs that are responsible for acceptor saccharide binding and product binding in yeast (Nagahashi et al., 1995; Choquer et al., 2004; Zakrzewski et al., 2014). These two motifs are well conserved in all classes of CHSs, confirming their critical roles. Interestingly, four functional specificity-determining sites are adjacent to these two motifs. The site 409 upstream three residues of the EDRXL motif underwent alteration in class III CHSs from Tyr (Y) to Phe (F). Likewise, the site 462 downstream six residues of the QRRRW motif underwent alteration in class III CHSs from Phe (F) to Ala (A). These sites may be responsible for functional divergence of classes III and the other three classes of division I.

\section{Members of Classes III and VIb CHS Genes are Specifically Up-Regulated during Infection}

Because classes III, VI $b$, and VIc were mainly retained or expanded in important pathogenic fungi, we examined the expression of these CHSs during infection. In the wheat scab fungus Fusarium graminearum, one of two paralogs of class III CHS genes (FGSG_10116) was specifically up-regulated during spike infection of barley and wheat (Figure 6). This class III gene had the highest expression change during host infection relative to other CHS genes, in agreement with the results of a recent study (Cheng et al., 2015). Deletion of this gene was lethal to F. graminearum (Cheng et al., 2015). Its ortholog in M. oryzae (MGG_01802) was responsible for virulence (Kong et al., 2012). In the stem rust fungus Puccinia graminis $\mathrm{f}$. sp. tritici, one of the three copies (PGTG_02527) of class III CHSs was also highly induced during infection of wheat and barley (Figure S5A). These 

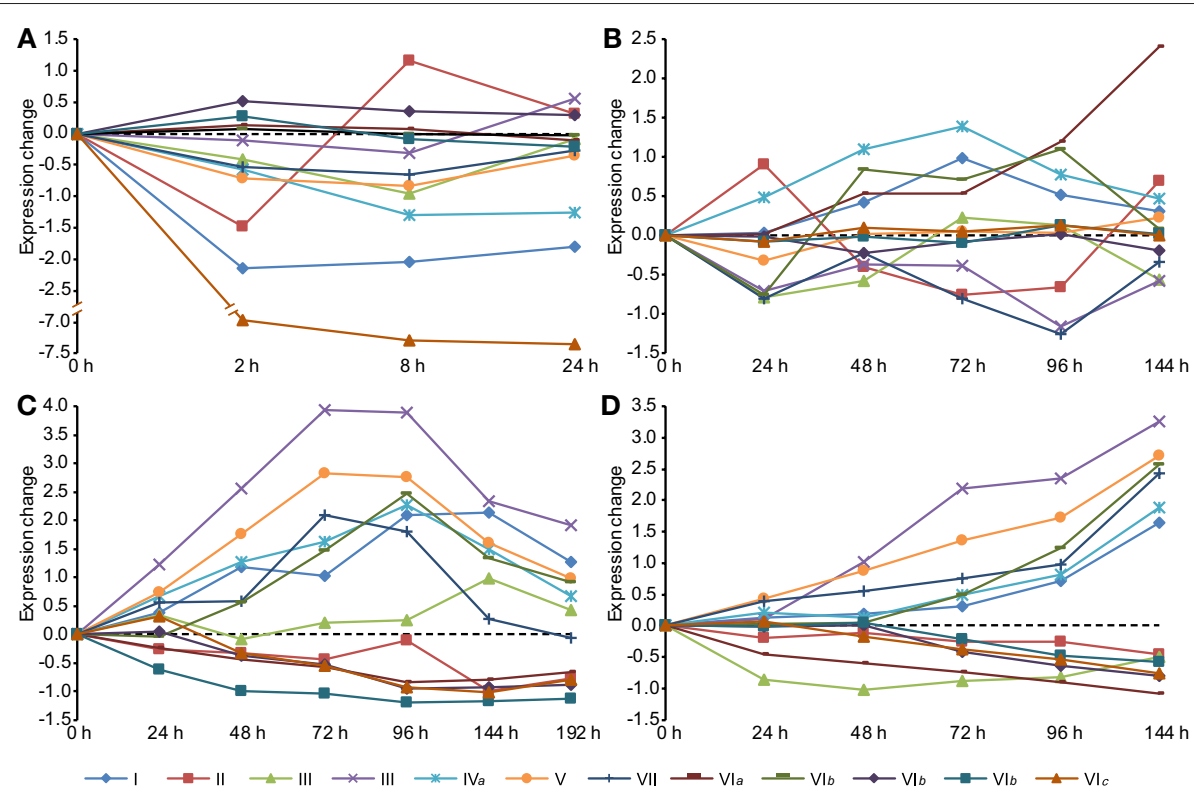

FIGURE 6 | Expression profiles of CHS genes during different stages in Fusarium graminearum. (A) Conidia germination. (B) Sexual development in vitro. (C) Infection of wheat. (D) Infection of barley spikes. In each condition, the mean values of RMA normalized expression data of three independent biological duplicates were analyzed. All time-series were transformed to $\left(0, v_{-} 1-v_{-} 0, v_{-} 1-v_{-} 0, \ldots, v_{-} n-v_{-} 0\right)$ so that the time series begins at 0 .

results indicate that the orthologous class III in pathogenic fungi may perform important functions during host infection.

One CHS gene of class VIb (FGSG_06550) from F. graminearum was highly induced during the later phases of infection (Figure 6). Its ortholog (GI: 310801203) in the maize anthracnose fungus Glomerella graminicola was also up-regulated during infection and had the highest expression level in the necrotrophic phase among all CHSs (Figure S5B), suggesting that this class VI $b$ CHS is important for pathogenesis.

In addition, genes in classes V (FGSG_01964) and VII (FGSG_12039) were highly induced during host infection. Previous studies have demonstrated that the classes V and/or VII CHSs are required for pathogenicity in many plant pathogens, such as F. graminearum, F. verticillioides, Fusarium oxysporum, M. oryzae, G. graminicola, and Ustilago maydis (Madrid et al., 2003; Garcerá-Teruel et al., 2004; Werner et al., 2007; MartínUrdíroz et al., 2008; Kim et al., 2009; Treitschke et al., 2010; Larson et al., 2011; Kong et al., 2012).

\section{The CHS-Associated Network is Conserved in Pathogenic Fungi}

To better understand the functional conservation of CHSs in pathogenic fungi, we compared functional protein-associated networks involved in chitin biosynthesis, constructed based on co-expression evidence and experimental evidence from the STRING database (Szklarczyk et al., 2015) in three important pathogenic fungi. A total of 57,39 , and 36 genes were determined to be functionally associated with the CHSs in F. graminearum, M. oryzae, and U. maydis, respectively (Figure 7A; Table S2). In the three CHS-associated networks, about half of the genes were conserved (Figures 7A,B). Gene ontology (GO) enrichment analysis showed that, beyond chitin biosynthesis, genes involved in ascospore formation, phenotypic switching, positive regulation of endocytosis, and response to salicylic acid were significantly enriched in these conserved orthologous genes (Figure 7C; Table S3). These results indicated that the CHS-associated network is likely related to fungal pathogenicity. In F. graminearum, ascospores are the primary inoculum for infecting wheat and barley (Trail et al., 2002). In addition, salicylic acid (SA) is considered to be a key plant defense hormone. A previous study demonstrated that $U$. maydis could sense and restrict the SA-levels of host plants to assure colonization success (Rabe et al., 2013).

We identified the putative protein complexes or parts of pathways hidden in the CHS network of $F$. graminearum constructed from the evidence of genomic context, experiments and text-mining. Among the 171 genes in the network, six interaction clusters were detected and found to be comprised of 20, 34, 17, 7, 5, and 3 genes, respectively (Figure 8A; Table S4). Based on the expression data downloaded from PLEXdb (Dash et al., 2012), most of the genes in these clusters were up-regulated during the later phases $(72,96$, or $144 \mathrm{~h})$ of wheat infection. Although some genes exhibited the opposite expression pattern, the entire clusters seemed to be co-regulated. For example, contrary to most genes in cluster 2 that were induced along the timeline, five genes in the same cluster were repressed (Figure 8B). These results suggested that genes in these clusters might play important roles in the necrotrophic phase of $F$. graminearum. Further functional enrichment analysis indicated that genes in cluster 2 are involved in various processes, such as pathogenesis, the MAPK cascade, small GTPase-mediated signal transduction, transmembrane receptor protein serine/threonine 


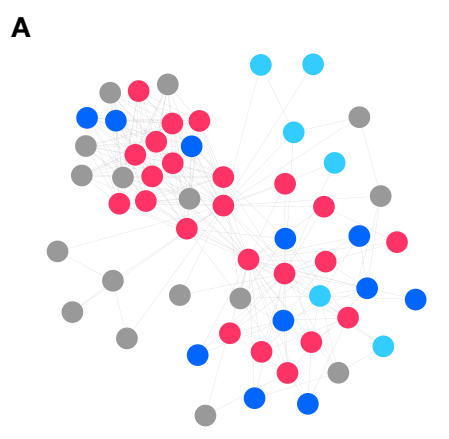

$\mathrm{Fg}$

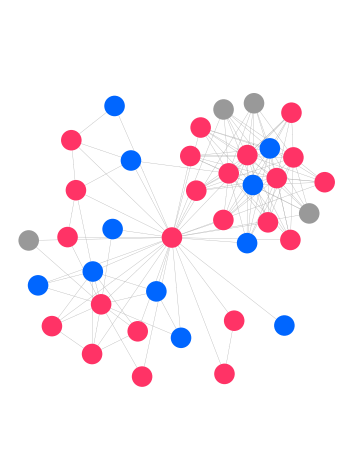

Mo

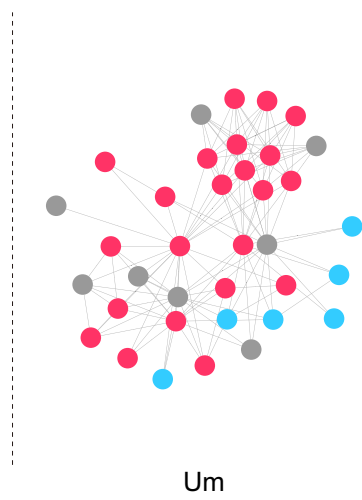

Um

Orthologs found in: • All networks • Networks of Fg \& Mo Networks of Fg \& Um • Only one network

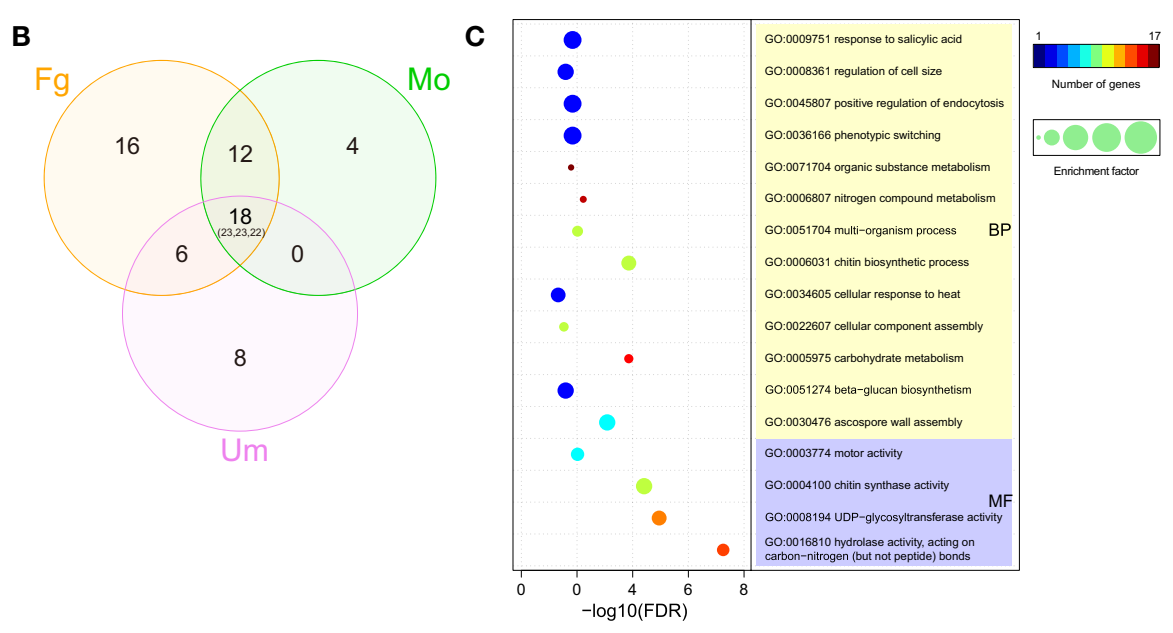

FIGURE 7 | CHS-associated networks in Fusarium graminearum, Magnaporthe oryzae, and Ustilago maydis. (A) CHS-associated networks in three plant pathogens. Nodes and edges represent genes and functional links, respectively. Node colors indicate the range of ortholog groups that appear in three networks. (B) Venn diagram for the distribution of ortholog groups in the three networks. Numbers correspond to ortholog groups, while the numbers in brackets represent the total number of genes (Ordering: Fg-Mo-Um). (C) Selected GO terms enriched in ortholog genes that are conserved in the three networks. The circle colors indicate the number of genes with a relevant GO term in the test set. The circle size indicates the ratio of the percentage of genes with relevant GO terms in the test set to that in the reference set (enrichment factor). Fg, F. graminearum; Mo, M. oryzae; Um, U. maydis. Please see Tables S2, S3 for more detailed information.

kinase signaling pathway, and ascospore formation (Figure 8C; Table S5). Many of these processes are related to fungal pathogenicity (Bölker, 1998; Xu et al., 1998; Beyer and Verreet, 2005). In addition, cluster I includes acetyl-CoA carboxylase and aspartate carbamoyltransferase, which are essential for the survival of fungi due to their crucial roles in the biosynthesis of fatty acids and pyrimidines (Simmer et al., 1990; Tong, 2005). Furthermore, calcineurin subunit B (FGSG_07404) in cluster 4 is involved in the adaptation to pheromone during conjugation with cellular fusion, which is essential for normal vegetative growth in Neurospora crassa (Kothe and Free, 1998).

\section{CONCLUSIONS}

We systematically identified and compared CHS genes across 109 representative fungi from Ascomycota, Basidiomycota, Cryptomycota, Microsporidia, Chytridiomycota, Zygomycota, Neocallimastigomycota, Entomophthoramycotina, and Glomeromycota. Comparative analysis revealed diversity in the number of CHS genes among different fungal lineages. Phylogenetic analysis revealed that the fungal CHS gene family is comprised of at least 10 ancestral orthologous clades, which have undergone frequent duplications and losses in different fungal lineages during evolution. Our results showed that CHS genes in classes III and VI $b$ have expanded or have been retained in pathogenic fungi and that they likely perform important functions during host infection. Comparative analysis revealed that CHS-associated networks are conserved among important pathogenic fungi, and participate in various important processes, including sexual reproduction and plant infection. In addition, many specificity-determining sites are located at or adjacent to important sites such as ligand binding and acceptor saccharide sites of CHSs and probably lead to functional diversification in the CHS gene family. These specificity-determining sites may be attractive targets for further structural and experimental studies. Results from this study elucidated orthologous and paralogous relationships among various CHS genes and provided new insights into the evolution and function of the fungal CHS genes. 

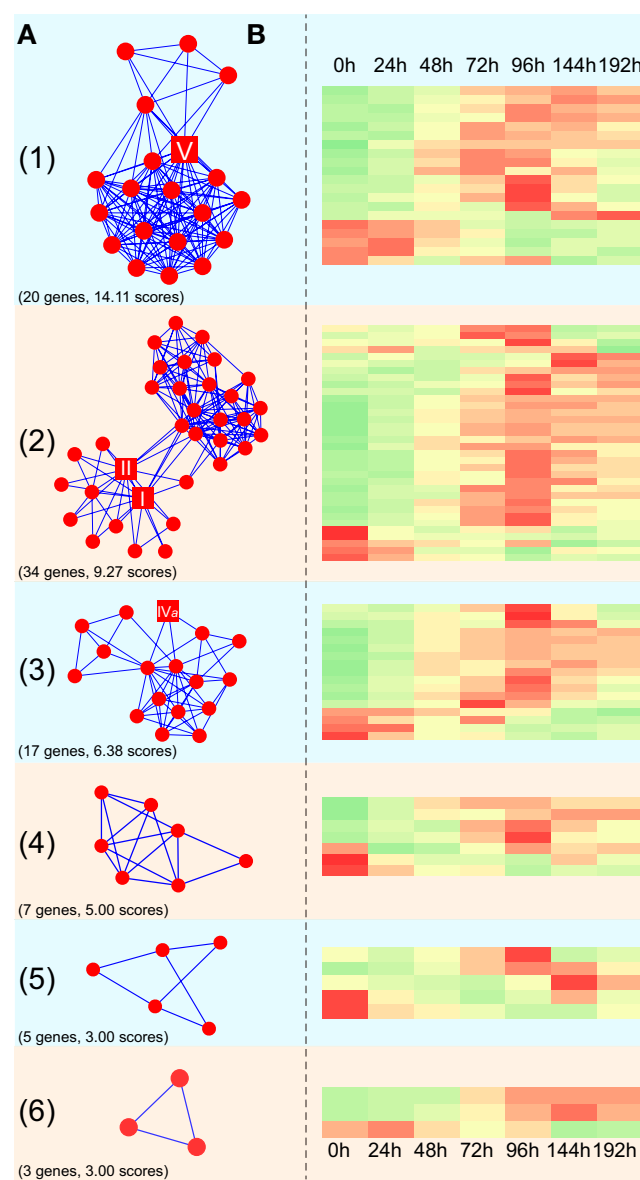

C

(1) protein serine/threonine kinase activity;

(2) transmembrane transporter activity;

(3) acetyl-CoA carboxylase activity;

(4) aspartate carbamoyltransferase activity.

(1) pathogenesis; (2) activation of MAPK activity;

(3) small GTPase mediated signal transduction;

(4) transmembrane receptor protein serine/

threonine kinase signaling pathway;

(5) conidium \& ascospore formation;

(6) chitin biosynthetic process.

(1) response to salicylic acid;

(2) small GTPase mediated signal transduction;

(3) cell morphogenesis; (4) ascospore formation;

(5) phenotypic switching.

(1) response to pheromone involved in conjugation with cellular fusion;

(2) chitin biosynthetic process.

(1) small GTPase mediated signal transduction;

(2) organelle organization.

NA

FIGURE 8 | Expression profiles of interaction clusters in CHS-associated networks in $\boldsymbol{F}$ graminearum. (A) Interaction clusters identified in the network of $\mathrm{CHSs}$ in F. graminearum. The square nodes represent CHSs of $F$. graminearum. Numbers in the brackets correspond to gene numbers and scores calculated by MCODE (Bader and Hogue, 2003), respectively. Please see Table S4 for more detailed information. (B) Dynamic expression profiling of interaction clusters during infection of wheat. Each row in the heatmap indicates a single gene and each column represents a time point after wheat was inoculated with $F$. graminearum. The scale bar represents the Z-scores of $\log _{2}$-fold change. (C) Selected GO terms enriched in five interaction clusters. Please see Table S5 for more detailed information.

\section{MATERIALS AND METHODS}

\section{Collection of Fungal Genomes}

The predicted proteomes of 109 public fungal genomes (Table S1) were collected from GenBank of National Center for Biotechnology Information (NCBI), Joint Genome Institute (JGI) site of DOE (http://genome.jgi.doe.gov/programs/fungi/ index.jsf), Fungal Genome Initiative (FGI) site of Broad Institute (http://www.broadinstitute.org/science/projects/projects/ fungal-genome-initiative), UniProt database (http://www. uniprot.org/proteomes/), and Blugen (http://www.blugen.org/).

\section{Identification of Putative CHSs in Fungi}

The Hmmscan program in HMMER 3.0 package (Eddy, 2011) was used to search each of the fungal proteomes with the domain-specific HMM profiles of chitin synthases downloaded from the Pfam database (Finn et al., 2014) as queries, including Chitin_synth_1 (PF01644) and Chitin_synth_2 (PF03142) domains. The primary result was filtered with the score of 20 as the cutoff. Only the sequences marked as CHSs (best match) were submitted to the Pfam database and the SMART database (Letunic et al., 2012) to confirm the chitin synthase domains.

\section{Sequence Alignment and Phylogenetic Analysis}

Multiple sequence alignments were generated with the M-Coffee program (Di Tommaso et al., 2011) using the combination of TCoffee, MAFFT, MUSCLE, and ProbCons methods and further edited manually. CHSs in division I were aligned based on the continuous CS1N, CS1, and CS2 domains, whereas CHSs in divisions II and III were aligned based only on the CS2 domain. Phylogenies were subsequently constructed by the Maximum likelihood (ML) method using PhyML3.1 (Guindon et al., 2010) with eight categories of $\gamma$-distributed substitution rates and SPRs algorithms, based on the best-fit model LG + G estimated by ProtTest2.4 (Abascal et al., 2005). SH-like approximate likelihood ratios (aLRT-SH) supports were used to evaluate the reliability of internal branches. The trees were further edited using the ITOL 
tool (Letunic and Bork, 2007). The identity scores of alignment were extracted with BioEdit software and then the heat map was constructed with the gplot package in $\mathrm{R}$.

\section{Prediction of Specificity-Determining Sites in the Fungal CHS Gene Family}

Complete sequences of CHSs in each division were aligned using COBALT (Papadopoulos and Agarwala, 2007). Specificitydetermining sites were identified using SPEER-SERVER (http:// www.hpppi.iicb.res.in/ss/index.html) with highest scores. The options of $20 \%$ gap allowed per column and a weight of 1.0 for relative entropy and physico-chemical (PC) properties were used (Chakraborty et al., 2012). A sequence logo of each class of CHSs was created with WebLogo 3 (Crooks et al., 2004). Structural sites and functional sites were identified using the ConSurf Server (Ashkenazy et al., 2010). The default Bayesian calculation method and JTT evolutionary substitution model were used.

\section{Analysis of Gene Expression Profiles of CHSs in Plant Pathogens}

The mean values of Robust Multi-array Average (RMA) normalized expression data of F. graminearum were obtained from the Plant Expression Database (PLEXdb) (http://www. plexdb.org/index.php) with four individual experiments, including conidia germination (Experiment FG7), sexual development (Experiment FG5) and spike infection of barley (Experiment FG1) and wheat (Experiment FG15). For each experiment, all time-series were transformed to $\left(0, v_{-} \_1-v_{-} 0, \ldots\right.$, v_n-v_0) so that the time series begins at 0 . The normalized gene expression data of G. graminicola (O'Connell et al., 2012) and P. graminis f. sp. tritici (Duplessis et al., 2011) were downloaded from NCBI GEO (accession no. GSE34632 and GSE25020). The data of three biological replicates were averaged and further transformed to $\log _{2}$ ratios.

\section{Network Analysis}

We used CHSs as baits to extract all the functional proteinassociated networks using different evidence in the STRING

\section{REFERENCES}

Abascal, F., Zardoya, R., and Posada, D. (2005). ProtTest: selection of best-fit models of protein evolution. Bioinformatics 21, 2104-2105. doi: 10.1093/bioinformatics/bti263

Ashkenazy, H., Erez, E., Martz, E., Pupko, T., and Ben-Tal, N. (2010). ConSurf 2010: calculating evolutionary conservation in sequence and structure of proteins and nucleic acids. Nucleic Acids Res. 38, W529-W533. doi: 10.1093/nar/gkq399

Bader, G. D., and Hogue, C. W. (2003). An automated method for finding molecular complexes in large protein interaction networks. $B M C$ Bioinformatics 4:2. doi: 10.1186/1471-2105-4-2

Bauer, S., Grossmann, S., Vingron, M., and Robinson, P. N. (2008). Ontologizer 2.0-a multifunctional tool for GO term enrichment analysis and data exploration. Bioinformatics 24, 1650-1651. doi: 10.1093/bioinformatics/btn250

Beyer, M., and Verreet, J. A. (2005). Germination of Gibberella zeae ascospores as affected by age of spores after discharge and environmental factors. Eur. J. Plant Pathol. 111, 381-389. doi: 10.1007/s10658-004-6470-9 (version 10) database (Szklarczyk et al., 2015) with a confidence score of 0.4. Then CHS-associated networks were visualized in Cytoscape (version 3.2.0) software (Shannon et al., 2003). Interaction clusters were detected using MCODE (version 1.4.1) plugin (Bader and Hogue, 2003) with the default parameters. Functional enrichment analysis was performed using Ontologizer 2.1 software (Bauer et al., 2008) with the whole $F$. graminearum annotation as the reference set. The term-for-term approach and Benjamini and Hochberg FDR correction (adjusted $p<0.05)$ options were used. Heatmaps were constructed with the R package gplot. Hierarchical clustering was carried out with Pearson correlation distance measure and the pairwise averagelinkage method.

\section{AUTHOR CONTRIBUTIONS}

JRX conceived the study; HL designed and coordinated the study; ML and CJ performed bioinformatic analyses; QW and ZZ contributed to the data collection; ML, CJ, QW, QJ, JX, and HL interpreted the results; ML and HL wrote the paper. All authors read, corrected and approved the final manuscript.

\section{ACKNOWLEDGMENTS}

We thank Dr. Larry Dunkle at Purdue University for proofreading. This work was supported by the National Major Project of Breeding for New Transgenic Organisms (2012ZX08009003), grant 2013CB127702 from the National Basic Research Program of China (973 program) and the Special Fund for Agro-scientific Research in the Public Interest (201303016).

\section{SUPPLEMENTARY MATERIAL}

The Supplementary Material for this article can be found online at: http://journal.frontiersin.org/article/10.3389/fpls.2016. 00037

Bölker, M. (1998). Sex and crime: heterotrimeric G proteins in fungal mating and pathogenesis. Fungal Genet. Biol. 25, 143-156. doi: 10.1006/fgbi.1998.1102

Bowman, S. M., and Free, S. J. (2006). The structure and synthesis of the fungal cell wall. Bioessays 28, 799-808. doi: 10.1002/bies.20441

Cabib, E., Sburlati, A., Bowers, B., and Silverman, S. J. (1989). Chitin synthase 1, an auxiliary enzyme for chitin synthesis in Saccharomyces cerevisiae. J. Cell Biol. 108, 1665-1672. doi: 10.1083/jcb.108.5.1665

Chakraborty, A., Mandloi, S., Lanczycki, C. J., Panchenko, A. R., and Chakrabarti, S. (2012). SPEER-SERVER: a web server for prediction of protein specificity determining sites. Nucleic Acids Res. 40, W242-W248. doi: 10.1093/nar/gks559

Cheng, W., Song, X.-S., Li, H.-P., Cao, L.-H., Sun, K., Qiu, X.-L., et al. (2015). Host-induced gene silencing of an essential chitin synthase gene confers durable resistance to Fusarium head blight and seedling blight in wheat. Plant Biotechnol. J. 13, 1335-1345. doi: 10.1111/pbi.12352

Choquer, M., Boccara, M., Gonçalves, I. R., Soulié, M. C., and Vidal-Cros, A. (2004). Survey of the Botrytis cinerea chitin synthase multigenic family through the analysis of six euascomycetes genomes. Eur. J. Biochem. 271, 2153-2164. doi: 10.1111/j.1432-1033.2004.04135.x 
Crooks, G. E., Hon, G., Chandonia, J. M., and Brenner, S. E. (2004). WebLogo: a sequence logo generator. Genome Res. 14, 1188-1190. doi: 10.1101/gr.849004

Dash, S., Van Hemert, J., Hong, L., Wise, R. P., and Dickerson, J. A. (2012). PLEXdb: gene expression resources for plants and plant pathogens. Nucleic Acids Res. 40, D1194-D1201. doi: 10.1093/nar/gkr938

Di Tommaso, P., Moretti, S., Xenarios, I., Orobitg, M., Montanyola, A., Chang, J. M., et al. (2011). T-Coffee: a web server for the multiple sequence alignment of protein and RNA sequences using structural information and homology extension. Nucleic Acids Res. 39, W13-W17. doi: 10.1093/nar/gkr245

Duplessis, S., Cuomo, C. A., Lin, Y. C., Aerts, A., Tisserant, E., VeneaultFourrey, C., et al. (2011). Obligate biotrophy features unraveled by the genomic analysis of rust fungi. Proc. Natl. Acad. Sci. U.S.A. 108, 9166-9171. doi: 10.1073/pnas.1019315108

Eddy, S. R. (2011). Accelerated Profile HMM Searches. PLoS Comput. Biol. 7:e1002195. doi: 10.1371/journal.pcbi.1002195

Finn, R. D., Bateman, A., Clements, J., Coggill, P., Eberhardt, R. Y., Eddy, S. R., et al. (2014). Pfam: the protein families database. Nucleic Acids Res. 42, D222-D230. doi: 10.1093/nar/gkt1223

Free, S. J. (2013). Fungal cell wall organization and biosynthesis. Adv. Genet. 81, 33-82. doi: 10.1016/B978-0-12-407677-8.00002-6

Garcerá-Teruel, A., Xoconostle-Cázares, B., Rosas-Quijano, R., Ortiz, L., LeonRamirez, C., Specht, C. A., et al. (2004). Loss of virulence in Ustilago maydis by Umchs6 gene disruption. Res. Microbiol. 155, 87-97. doi: 10.1016/j.resmic.2003.11.005

$\mathrm{Gu}$, X. (2001). Maximum-likelihood approach for gene family evolution under functional divergence. Mol. Biol. Evol. 18, 453-464. doi: 10.1093/oxfordjournals.molbev.a003824

Guindon, S., Dufayard, J. F., Lefort, V., Anisimova, M., Hordijk, W., and Gascuel, O. (2010). New algorithms and methods to estimate maximum-likelihood phylogenies: assessing the performance of PhyML 3.0. Syst. Biol. 59, 307-321. doi: $10.1093 /$ sysbio/syq010

Kappes, F., Scholten, I., Richter, N., Gruss, C., and Waldmann, T. (2004). Functional domains of the ubiquitous chromatin protein DEK. Mol. Cell. Biol. 24, 6000-6010. doi: 10.1128/MCB.24.13.6000-6010.2004

Kim, J. E., Lee, H. J., Lee, J., Kim, K. W., Yun, S. H., Shim, W. B., et al. (2009). Gibberella zeae chitin synthase genes, GzCHS5 and GzCHS7, are required for hyphal growth, perithecia formation, and pathogenicity. Curr. Genet. 55, 449-459. doi: 10.1007/s00294-009-0258-6

Kong, L. A., Yang, J., Li, G. T., Qi, L. L., Zhang, Y. J., Wang, C. F., et al. (2012). Different chitin synthase genes are required for various developmental and plant infection processes in the rice blast fungus Magnaporthe oryzae. PLoS Pathog. 8:e1002526. doi: 10.1371/journal.ppat.1002526

Koonin, E. V. (2005). Orthologs, paralogs, and evolutionary genomics. Annu. Rev. Genet. 39, 309-338. doi: 10.1146/annurev.genet.39.073003.114725

Kothe, G. O., and Free, S. J. (1998). Calcineurin subunit B is required for normal vegetative growth in Neurospora crassa. Fungal Genet. Biol. 23, 248-258. doi: 10.1006/fgbi.1998.1037

Larson, T. M., Kendra, D. F., Busman, M., and Brown, D. W. (2011). Fusarium verticillioides chitin synthases CHS5 and CHS7 are required for normal growth and pathogenicity. Curr. Genet. 57, 177-189. doi: 10.1007/s00294-011-0334-6

Latgé, J. P. (2007). The cell wall: a carbohydrate armour for the fungal cell. Mol. Microbiol. 66, 279-290. doi: 10.1111/j.1365-2958.2007.05872.x

Letunic, I., and Bork, P. (2007). Interactive Tree Of Life (iTOL): an online tool for phylogenetic tree display and annotation. Bioinformatics 23, 127-128. doi: 10.1093/bioinformatics/btl529

Letunic, I., Doerks, T., and Bork, P. (2012). SMART 7: recent updates to the protein domain annotation resource. Nucleic Acids Res. 40, D302-305. doi: 10.1093/nar/gkr931

Ma, L. J., Ibrahim, A. S., Skory, C., Grabherr, M. G., Burger, G., Butler, M., et al. (2009). Genomic analysis of the basal lineage fungus Rhizopus oryzae reveals a whole-genome duplication. PLoS Genet. 5:e1000549. doi: 10.1371/journal.pgen.1000549

Madrid, M. P., Di Pietro, A., and Roncero, M. I. (2003). Class V chitin synthase determines pathogenesis in the vascular wilt fungus Fusarium oxysporum and mediates resistance to plant defence compounds. Mol. Microbiol. 47, 257-266. doi: 10.1046/j.1365-2958.2003.03299.x

Mandel, M. A., Galgiani, J. N., Kroken, S., and Orbach, M. J. (2006). Coccidioides posadasii contains single chitin synthase genes corresponding to classes I to VII. Fungal Genet. Biol. 43, 775-788. doi: 10.1016/j.fgb.2006. 05.005

Martín-Urdíroz, M., Roncero, M. I., González-Reyes, J. A., and Ruiz-Roldán, C. (2008). ChsVb, a class VII chitin synthase involved in septation, is critical for pathogenicity in Fusarium oxysporum. Eukaryot Cell 7, 112-121. doi: 10.1128/EC.00347-07

Merzendorfer, H. (2011). The cellular basis of chitin synthesis in fungi and insects: common principles and differences. Eur. J. Cell Biol. 90, 759-769. doi: 10.1016/j.ejcb.2011.04.014

Munro, C. A., and Gow, N. A. (2001). Chitin synthesis in human pathogenic fungi. Med. Mycol. 39(Suppl. 1), 41-53. doi: 10.1080/mmy.39.1.41.53

Nagahashi, S., Sudoh, M., Ono, N., Sawada, R., Yamaguchi, E., Uchida, Y., et al. (1995). Characterization of chitin synthase 2 of Saccharomyces cerevisiae. Implication of two highly conserved domains as possible catalytic sites. J. Biol. Chem. 270, 13961-13967. doi: 10.1074/jbc.270.23.13961

Niño-Vega, G. A., Carrero, L., and San-Blas, G. (2004). Isolation of the CHS4 gene of Paracoccidioides brasiliensis and its accommodation in a new class of chitin synthases. Med. Mycol. 42, 51-57. doi: 10.1080/1369378031000153811

O'Connell, R. J., Thon, M. R., Hacquard, S., Amyotte, S. G., Kleemann, J., Torres, M. F., et al. (2012). Lifestyle transitions in plant pathogenic Colletotrichum fungi deciphered by genome and transcriptome analyses. Nat. Genet. 44, 1060-1065. doi: 10.1038/ng.2372

Odenbach, D., Thines, E., Anke, H., and Foster, A. J. (2009). The Magnaporthe grisea class VII chitin synthase is required for normal appressorial development and function. Mol. Plant Pathol. 10, 81-94. doi: 10.1111/j.13643703.2008.00515.x

Pacheco-Arjona, J. R., and Ramirez-Prado, J. H. (2014). Large-scale phylogenetic classification of fungal chitin synthases and identification of a putative cellwall metabolism gene cluster in Aspergillus genomes. PLoS ONE 9:e104920. doi: 10.1371/journal.pone.0104920

Papadopoulos, J. S., and Agarwala, R. (2007). COBALT: constraint-based alignment tool for multiple protein sequences. Bioinformatics 23, 1073-1079. doi: 10.1093/bioinformatics/btm076

Rabe, F., Ajami-Rashidi, Z., Doehlemann, G., Kahmann, R., and Djamei, A. (2013). Degradation of the plant defence hormone salicylic acid by the biotrophic fungus Ustilago maydis. Mol. Microbiol. 89, 179-188. doi: 10.1111/mmi. 12269

Reese, T. A., Liang, H. E., Tager, A. M., Luster, A. D., Van Rooijen, N., Voehringer, D., et al. (2007). Chitin induces accumulation in tissue of innate immune cells associated with allergy. Nature 447, 92-U97. doi: 10.1038/nature05746

Roncero, C. (2002). The genetic complexity of chitin synthesis in fungi. Curr. Genet. 41, 367-378. doi: 10.1007/s00294-002-0318-7

Ruiz-Herrera, J., and Ortiz-Castellanos, L. (2010). Analysis of the phylogenetic relationships and evolution of the cell walls from yeasts and fungi. FEMS Yeast Res. 10, 225-243. doi: 10.1111/j.1567-1364.2009.00589.x

Schenkman, J. B., and Jansson, I. (2003). The many roles of cytochrome b5. Pharmacol. Ther. 97, 139-152. doi: 10.1016/S0163-7258(02)00327-3

Schmidt, M. (2004). Survival and cytokinesis of Saccharomyces cerevisiae in the absence of chitin. Microbiology 150, 3253-3260. doi: 10.1099/mic.0.27197-0

Schuster, M., Treitschke, S., Kilaru, S., Molloy, J., Harmer, N. J., and Steinberg, G. (2012). Myosin-5, kinesin-1 and myosin-17 cooperate in secretion of fungal chitin synthase. EMBO J. 31, 214-227. doi: 10.1038/emboj.2011.361

Shannon, P., Markiel, A., Ozier, O., Baliga, N. S., Wang, J. T., Ramage, D., et al. (2003). Cytoscape: a software environment for integrated models of biomolecular interaction networks. Genome Res. 13, 2498-2504. doi: 10.1101/gr.1239303

Shaw, J. A., Mol, P. C., Bowers, B., Silverman, S. J., Valdivieso, M. H., Durán, A., et al. (1991). The function of chitin synthases 2 and 3 in the Saccharomyces cerevisiae cell cycle. J. Cell Biol. 114, 111-123. doi: 10.1083/jcb.114.1.111

Shibuya, N., and Minami, E. (2001). Oligosaccharide signalling for defence responses in plant. Physiol. Mol. Plant Pathol. 59, 223-233. doi: 10.1006/pmpp.2001.0364

Silverman, S. J., Sburlati, A., Slater, M. L., and Cabib, E. (1988). Chitin synthase 2 is essential for septum formation and cell division in Saccharomyces cerevisiae. Proc. Natl. Acad. Sci. U.S.A. 85, 4735-4739. doi: 10.1073/pnas.85.13.4735

Simmer, J. P., Kelly, R. E., Rinker, A. G. Jr., Zimmermann, B. H., Scully, J. L., Kim, H., et al. (1990). Mammalian dihydroorotase: nucleotide sequence, peptide sequences, and evolution of the dihydroorotase domain of the 
multifunctional protein CAD. Proc. Natl. Acad. Sci. U.S.A. 87, 174-178. doi: 10.1073/pnas.87.1.174

Szklarczyk, D., Franceschini, A., Wyder, S., Forslund, K., Heller, D., HuertaCepas, J., et al. (2015). STRING v10: protein-protein interaction networks, integrated over the tree of life. Nucleic Acids Res. 43, D447-D452. doi: $10.1093 / \mathrm{nar} / \mathrm{gku} 1003$

Tong, L. (2005). Acetyl-coenzyme A carboxylase: crucial metabolic enzyme and attractive target for drug discovery. Cell. Mol. Life Sci. 62, 1784-1803. doi: 10.1007/s00018-005-5121-4

Trail, F., Xu, H., Loranger, R., and Gadoury, D. (2002). Physiological and environmental aspects of ascospore discharge in Gibberella zeae (anamorph Fusarium graminearum). Mycologia 94, 181-189. doi: 10.2307/3761794

Treitschke, S., Doehlemann, G., Schuster, M., and Steinberg, G. (2010). The myosin motor domain of fungal chitin synthase $\mathrm{V}$ is dispensable for vesicle motility but required for virulence of the maize pathogen Ustilago maydis. Plant Cell 22, 2476-2494. doi: $10.1105 /$ tpc. 110.075028

Tsuizaki, M., Takeshita, N., Ohta, A., and Horiuchi, H. (2009). Myosin motorlike domain of the class VI chitin synthase CsmB is essential to its functions in Aspergillus nidulans. Biosci. Biotechnol. Biochem. 73, 1163-1167. doi: 10.1271/bbb. 90074

Werner, S., Sugui, J. A., Steinberg, G., and Deising, H. B. (2007). A chitin synthase with a myosin-like motor domain is essential for hyphal growth, appressorium differentiation, and pathogenicity of the maize anthracnose fungus Colletotrichum graminicola. Mol. Plant Microbe Interact. 20, 1555-1567. doi: 10.1094/MPMI-20-12-1555

Xu, J. R., Staiger, C. J., and Hamer, J. E. (1998). Inactivation of the mitogenactivated protein kinase Mps1 from the rice blast fungus prevents penetration of host cells but allows activation of plant defense responses. Proc. Natl. Acad. Sci. U.S.A. 95, 12713-12718. doi: 10.1073/pnas.95.21.12713

Zakrzewski, A. C., Weigert, A., Helm, C., Adamski, M., Adamska, M., Bleidorn, C., et al. (2014). Early divergence, broad distribution, and high diversity of animal chitin synthases. Genome Biol. Evol. 6, 316-325. doi: 10.1093/gbe/ evu011

Conflict of Interest Statement: The authors declare that the research was conducted in the absence of any commercial or financial relationships that could be construed as a potential conflict of interest.

Copyright $\odot 2016$ Li, Jiang, Wang, Zhao, Jin, Xu and Liu. This is an open-access article distributed under the terms of the Creative Commons Attribution License (CC $B Y)$. The use, distribution or reproduction in other forums is permitted, provided the original author(s) or licensor are credited and that the original publication in this journal is cited, in accordance with accepted academic practice. No use, distribution or reproduction is permitted which does not comply with these terms. 